\title{
BEHAVIOR OF SOLUTIONS OF BURGERS'S EQUATION WITH NONLOCAL BOUNDARY CONDITIONS (II)
}

By

\section{KENG DENG}

University of Southwestern Louisiana, Lafayette, Louisiana

Abstract. We study the large-time behavior of positive solutions of Burgers's equation $u_{t}=u_{x x}+\varepsilon u u_{x}, 0<x<1, t>0(\varepsilon>0)$, subject to the nonlocal boundary condition: $\quad-u_{x}(0, t)-\frac{1}{2} \varepsilon u^{2}(0, t)=a u^{p}(0, t)\left(\int_{0}^{1} u(x, t) d x\right)^{q}, u(1, t)=0$ $(0<p, q<\infty)$. The steady-state problem is analyzed in detail, and the result about finite-time blow-up is proved.

1. Introduction. In this paper, we are concerned with the following initial nonlocal boundary-value problem:

$$
\begin{aligned}
& u_{t}=u_{x x}+(f(u))_{x}, \quad 0<x<1, \quad t>0, \\
& -u_{x}(0, t)=g(u(0, t), \bar{u}), \quad t>0 \text {, } \\
& u(1, t)=0, \quad t>0, \\
& u(x, 0)=u_{0}(x) ; \quad 0 \leq x \leq 1,
\end{aligned}
$$

here $f$ and $g$ are continuously differentiable functions satisfying $f(0)=0$ and $g(0, v)=0, \bar{u}=\int_{0}^{1} u(x, t) d x$, and $u_{0}(x)$ is a nonnegative prescribed function. We are primarily interested in the power law cases $f(u)=\frac{1}{2} \varepsilon u^{2} \quad(\varepsilon>0), g(u, v)=$ $a u^{p} v^{q}$ or $g(u, v)=a u^{p} v^{q}+f(u)(p, q>0)$. We shall give the bifurcation diagrams for the stationary solutions and present stability-instability results.

In an earlier paper [1], we consider a problem closely related to (B) as follows:

$$
\begin{aligned}
& u_{t}=u_{x x}+(f(u))_{x}, \quad 0<x<1, \quad t>0, \\
& u(0, t)=0, \quad t>0, \\
& u_{x}(1, t)=g(u(1, t), \bar{u}), \quad t>0 \text {, } \\
& u(x, 0)=u_{0}(x), \quad 0 \leq x \leq 1,
\end{aligned}
$$

where the model problems are $f(u)=\frac{1}{2} \varepsilon u^{2}$ and $g(u, v)=a u^{p} v^{q}$ or $g(u, v)=$ $a u^{p} v^{q}-f(u)$. 
When $\varepsilon=0,(A)$ and (B) are essentially the same. However, for positve $\varepsilon$, notable differences can be observed. From a physical point of view, the convective term, $\frac{1}{2} \varepsilon u^{2}$, acts toward the left boundary and $-\left(u_{x}+\frac{1}{2} \varepsilon u^{2}\right)$ represents the flux. Therefore, for problem (A) the nonlocal forcing at the boundary is in the same direction as the effects of convection, while for problem (B) the flow through the boundary is in opposition to the effects of convection. Such physical contrast results in quite different diagrams, and consequently, there exists a clear distinction in dynamical analysis.

The plan of this paper will follow that of [1]. In the second section we establish the characterization of positive solutions of Burgers's equation. In Sec. 3 we show that some solutions blow up in finite time. Then we obtain the solution diagrams and present the stability results for Burgers's equation in the last section. Because many of the proofs bear much similarity to their counterparts in [1,3], we shall mainly state the theorems for problem (B) unless there is technical necessity. It should be pointed out that some problems remain unsolved. For instance, in Burgers's equation with $g(u, v)=a u^{p} v^{q}+\frac{1}{2} \varepsilon u^{2}$, our results partly rely on numerical experiments. However, all results for purely local boundary conditions with $g(u)=a u^{p}$ in [3] are covered. Furthermore, it is worth noting that the solution diagrams for Burgers's equation with $g(u, v)=a u^{p} v^{q}+\frac{1}{2} \varepsilon u^{2}$ are opposite those with $g(u, v)=a u^{p} v^{q}$.

2. Stationary solutions and their basic properties. In this section, we focus on the stationary solutions of $(B)$, which solve the problem:

$$
\begin{gathered}
w^{\prime \prime}(x)+(f(w))^{\prime}=0, \quad 0<x<1, \\
-w^{\prime}(0)=g(w(0), \bar{w}), \quad w(1)=0 .
\end{gathered}
$$

Here, $\bar{w}=\int_{0}^{1} w(x) d x$.

We first state

Lemma 2.1. Nontrivial solutions of $\left(B_{1}\right)$ are of one sign. Moreover, every positive solution $w(x)$ of $\left(\mathrm{B}_{1}\right)$ satisfies $w^{\prime}(x)<0$ on $[0,1]$.

Placing an additional restriction on $f$, we then have

LEMMA 2.2. If $f$ is twice continuously differentiable, $f^{\prime}(u)$ is strictly increasing for $u>0$, and $w_{1}(x), w_{2}(x)$ are two positive solutions of $\left(\mathbf{B}_{1}\right)$ that satisfy $w_{1}(0)>$ $w_{2}(0)$; then $w_{1}(x)>w_{2}(x)$ on $[0,1)$.

Proof. Let $w(x)=w_{1}(x)-w_{2}(x)$; then $w(x)$ satisfies

$$
\begin{gathered}
w^{\prime \prime}+f^{\prime}\left(w_{1}\right) w^{\prime}+\left(f^{\prime}\left(w_{1}\right)-f^{\prime}\left(w_{2}\right)\right) w_{2}^{\prime}=0, \quad 0<x<1, \\
w(0)>0 \text { and } w(1)=0 .
\end{gathered}
$$

Because $f^{\prime}(u)$ is strictly increasing and $w_{2}^{\prime}<0, w$ cannot have a negative interior minimum. If $w$ had a zero at $x_{0}$ in $(0,1)$, the maximum principle would yield $w \equiv 0$ on $\left[x_{0}, 1\right]$. Hence, $w^{\prime \prime}+f^{\prime}\left(w_{1}\right) w^{\prime}+f^{\prime \prime}(\xi) w_{2}^{\prime} w=0$ with $w\left(x_{0}\right)=w^{\prime}\left(x_{0}\right)=0$ implies that $w \equiv 0$ on $(0,1]$, which is impossible. 
By analogous reasoning as in [1], we obtain the following result:

THEOREM 2.3. Let $f^{\prime}$ be strictly increasing for $u>0$, and suppose that $g\left(u_{1}, v_{1}\right) / u_{1} \leq g\left(u_{2}, v_{2}\right) / u_{2}$ or $g\left(u_{1}, v_{1}\right) / v_{1} \leq g\left(u_{2}, v_{2}\right) / v_{2}$ for $u_{1}>u_{2}>0$ and $v_{1}>v_{2}>0$. Then, at most, one positive solution of $\left(\mathrm{B}_{1}\right)$ exists.

Finally we establish a relationship between solutions of $\left(B_{1}\right)$ and those from integral equations.

THEOREM 2.4. Let $f^{\prime}>0$ for $u>0$. Suppose that $w(x)$ is a positive solution of $\left(\mathrm{B}_{1}\right) \in C^{2}(0,1) \cap C^{1}[0,1]$. Then $w(x)$ satisfies

$$
G(w ; \alpha, \beta)=\int_{0}^{w(x)} \frac{d \sigma}{g(\alpha, \beta)-f(\alpha)+f(\sigma)}=1-x
$$

for $0 \leq x \leq 1$ and $g(\alpha, \beta)-f(\alpha)>0$. Here, $\alpha=w(0)$ and $\beta=\int_{0}^{1} w(x) d x$.

Conversely, if $\alpha>0$ satisfies $G(\alpha ; \alpha, \beta)=1$, and if positive $w(x)$ satisfies $G(w ; \alpha, \beta)=1-x$ with $\int_{0}^{1} w(x) d x=\beta$, and if $g(\alpha, \beta)-f(\alpha)>0$, then $w(x)$ is a positive solution of $\left(B_{1}\right)$.

Proof. This result is proved much like the parallel proof in [1]. However, we will include a sketch of the proof indicating where the condition $g(\alpha, \beta)-f(\alpha)>0$ is involved.

Let $w(x)$ be a solution of $\left(\mathbf{B}_{1}\right)$. Then a quadrature yields

$$
g(\alpha, \beta)-f(\alpha)=-w^{\prime}(x)-f(w(x))
$$

on $[0,1]$. In particular, $g(\alpha, \beta)-f(\alpha)=-w^{\prime}(1)$ which is positive by Lemma 2.1.

Conversely, if $g(\alpha, \beta)-f(\alpha)>0$, then $g(\alpha, \beta)-f(\alpha)+f(\sigma)$ is positive for $\sigma \in[0, \alpha]$. Thus the proof can proceed in the same manner as that in Theorem 2.4A of [3].

Making use of Theorem 2.4, we characterize the set of positive solutions of Burgers's equation. For problem $\left(\mathrm{B}_{1}\right)$ with $g(u, v)=a u^{p} v^{q}$, as in [1], the change of variables $y=\int_{0}^{x} w(s) d s$ and $h(y)=u^{2}(x(y))$ leads to

$$
\begin{aligned}
h(y) & =C^{2}\left(1-e^{-\varepsilon(\beta-y)}\right), \\
w(x) & =C\left(1-e^{-\varepsilon \beta+\varepsilon \int_{0}^{x} w(s) d s}\right),
\end{aligned}
$$

and

$$
w^{\prime}(x)=-\frac{1}{2} C^{2} \varepsilon e^{-\varepsilon \beta+\varepsilon \int_{0}^{x} w(s) d s} .
$$

Consequently, we find the following equation:

$$
\alpha^{p-2}=\frac{\varepsilon}{2 a}\left(e^{\varepsilon \beta}-1\right)^{-1} \beta^{q},
$$

which is the same as $(2.3)$ in [1]. We now need, however, an additional condition:

$$
\frac{2 a}{\varepsilon} \alpha^{p-2} \beta^{q}>1
$$


Taking this condition into account, we obtain

$$
G_{1}(\alpha, \beta)=\frac{1}{2} \varepsilon \alpha G(\alpha ; \alpha, \beta)=\int_{0}^{1} \frac{d \sigma}{\left(\frac{2 a}{\varepsilon}\right) \alpha^{p-2} \beta^{q}-1+\sigma^{2}}=\frac{1}{2} \varepsilon \alpha .
$$

For $p=2$, let $\beta^{*}=\left(\frac{\varepsilon}{2 a}\right)^{1 / q}$ and set $Q(\beta)=\frac{2 a}{\varepsilon} \beta^{q}, R(\beta)=\left(e^{\varepsilon \beta}-1\right)^{-1}$. We wish to solve $Q(\beta)=R(\beta)$. We have $Q\left(\beta^{*}\right)=1, \quad Q(+\infty)=+\infty$, and $Q^{\prime}(\beta)>0$ for $\beta$ in $\left(\beta^{*}, \infty\right) ; \quad R(\beta) \rightarrow 0$ as $\beta \rightarrow+\infty$, and if $\varepsilon<(2 a)^{\frac{1}{1+q}}(\ln 2)^{\frac{q}{1+q}}$, then $R\left(\beta^{*}\right)>1$. Clearly, $R^{\prime}(\beta)<0$ on $\left(\beta^{*}, \infty\right)$. Thus, when $\varepsilon<(2 a)^{\frac{1}{1+q}}(\ln 2)^{\frac{q}{1+q}}$, there is a unique solution of $\left(\mathrm{B}_{1}\right)$ for $p=2$.

For $p \neq 2$, substitution of $(2.1)$ in $(2.3)$ yields

$$
\int_{0}^{1} \frac{d \sigma}{\left(e^{\varepsilon \beta}-1\right)^{-1}-1+\sigma^{2}}=\frac{\varepsilon}{2}\left(\frac{\varepsilon}{2 a}\right)^{\frac{1}{p-2}}\left(e^{\varepsilon \beta}-1\right)^{-\frac{1}{p-2}} \beta^{-\frac{q}{p-2}}
$$

Let $\gamma^{2}=\left(e^{\varepsilon \beta}-1\right)^{-1}-1$. We then have

$$
\int_{0}^{1} \frac{d \sigma}{\gamma^{2}+\sigma^{2}}=\delta\left(\gamma^{2}+1\right)^{\frac{1}{p-2}}\left[\ln \left(\frac{\gamma^{2}+2}{\gamma^{2}+1}\right)\right]^{\frac{q}{2-p}} \quad \text { on }(0, \infty),
$$

where $\delta=\varepsilon^{\frac{p+q-1}{p-2}} 2^{\frac{1-p}{p-2}} a^{\frac{1}{2-p}}$.

Set

$$
\Psi(\gamma)=\left(\gamma^{2}+1\right)^{\frac{1}{2-p}}\left[\ln \left(\frac{\gamma^{2}+2}{\gamma^{2}+1}\right)\right]^{\frac{q}{p-2}} \int_{0}^{1} \frac{d \sigma}{\gamma^{2}+\sigma^{2}}=\delta .
$$

We then find that

$$
\Psi(\gamma)=\tan ^{-1} \frac{1}{\gamma} \gamma^{-1}\left(\gamma^{2}+1\right)^{\frac{1}{2-p}}\left[\ln \left(\frac{\gamma^{2}+2}{\gamma^{2}+1}\right)\right]^{-\frac{q}{2-p}} .
$$

If $p>2$, set $\lambda=\frac{1}{p-2}, \quad \mu=\frac{q}{p-2}$,

$$
\Psi(\gamma)=\tan ^{-1} \frac{1}{\gamma} \gamma^{-1}\left(\gamma^{2}+1\right)^{-\lambda}\left[\ln \left(\frac{\gamma^{2}+2}{\gamma^{2}+1}\right)\right]^{\mu},
$$

and

where

$$
\Psi^{\prime}(\gamma)=\tan ^{-1} \frac{1}{\gamma}\left(\gamma^{2}+1\right)^{-\lambda-1}\left[\ln \left(\frac{\gamma^{2}+2}{\gamma^{2}+1}\right)\right]^{\mu} J(\gamma),
$$

$$
J(\gamma)=-2 \lambda-\gamma^{-2}\left(\gamma^{2}+1\right)-\gamma^{-1} / \tan ^{-1} \frac{1}{\gamma}-\frac{2 \mu}{\gamma^{2}+2} / \ln \left(\frac{\gamma^{2}+2}{\gamma^{2}+1}\right) .
$$

Since $J(\gamma)<0$ on $(0, \infty)$, it follows that $\Psi^{\prime}(\gamma)<0$. We see that $\Psi(\gamma) \rightarrow+\infty$ as $\gamma \rightarrow 0^{+}$and that $\Psi(+\infty)=0$. Hence, $\left(B_{1}\right)$ has one and only one positive solution for each $\varepsilon>0, a>0$.

In the case of $p<2$, let $\lambda=\frac{1}{2-p}$ and $\mu=\frac{q}{2-p}$. We thus obtain

$$
\Psi(\gamma)=\tan ^{-1} \frac{1}{\gamma} \gamma^{-1}\left(\gamma^{2}+1\right)^{\lambda}\left[\ln \left(\frac{\gamma^{2}+2}{\gamma^{2}+1}\right)\right]^{-\mu}
$$


and

$$
\Psi^{\prime}(\gamma)=\tan ^{-1} \frac{1}{\gamma}\left(\gamma^{2}+1\right)^{\lambda-1}\left[\ln \left(\frac{\gamma^{2}+2}{\gamma^{2}+1}\right)\right]^{-\mu} J(\gamma),
$$

where

$$
J(\gamma)=2 \lambda-\gamma^{-2}\left(\gamma^{2}+1\right)-\gamma^{-1} / \tan ^{-1} \frac{1}{\gamma}+\frac{2 \mu}{\gamma^{2}+2} / \ln \left(\frac{\gamma^{2}+2}{\gamma^{2}+1}\right)
$$

and

$$
\begin{aligned}
J^{\prime}(\gamma)= & 2 \gamma^{-3}+\left[\gamma^{-2} / \tan ^{-1} \frac{1}{\gamma}-\frac{1}{\gamma\left(\gamma^{2}+1\right)} /\left(\tan ^{-1} \frac{1}{\gamma}\right)^{2}\right] \\
& +\left\{\frac{4 \mu \gamma}{\left(\gamma^{2}+2\right)^{2}\left(\gamma^{2}+1\right)} /\left[\ln \left(\frac{\gamma^{2}+2}{\gamma^{2}+1}\right)\right]^{2}-\frac{4 \mu \gamma}{\left(\gamma^{2}+2\right)^{2}} / \ln \left(\frac{\gamma^{2}+2}{\gamma^{2}+1}\right)\right\} \\
= & 2 \gamma^{-3}+J_{1}(\gamma)+J_{2}(\gamma) .
\end{aligned}
$$

Using $\tan ^{-1} x \geq \frac{x}{x^{2}+1}$ with $x=\gamma^{-1}$, we find that $J_{1}(\gamma) \geq 0$. We have $J_{2}(\gamma) \geq 0$ since $\ln (1+x) \leq x$ with $x=\frac{1}{\gamma^{2}+1}$. Thus, $J^{\prime}(\gamma)>0$ on $(0, \infty)$.

Since $1 \leq p<2$ corresponds to $1 \leq \lambda<\infty$, we observe that $J(\gamma) \rightarrow-\infty$ as $\gamma \rightarrow 0^{+}$and that $J(\gamma) \sim 2(\lambda+\mu+1)>0$ as $\gamma \rightarrow+\infty$. Hence $J(\gamma)$ changes sign only once, and it follows that none, one, or two positive solutions of $\left(B_{1}\right)$ exist, according to whether

$$
\varepsilon<\left(\Psi_{m}\right)^{\frac{2-p}{1-p-q}} 2^{\frac{1-p}{1-p-q}} a^{\frac{1}{p+q-1}}, \quad \varepsilon=\left(\Psi_{m}\right)^{\frac{2-p}{1-p-q}} 2^{\frac{1-p}{1-p-q}} a^{\frac{1}{p+q-1}}
$$

or

$$
\varepsilon>\left(\Psi_{m}\right)^{\frac{2-p}{1-p-q}} 2^{\frac{1-p}{1-p-q}} a^{\frac{1}{p+q-1}}
$$

where $\Psi_{m}=\min _{0<\gamma<\infty} \Psi(\gamma)$.

For $0<p<1, \frac{1}{2}<\lambda<1$. If $\lambda+\mu<1$, i.e., if $p+q<1, J(\gamma) \sim 2(\lambda+\mu-1)<0$ as $\gamma \rightarrow+\infty$; so $\Psi^{\prime}(\gamma)<0$ for any $\gamma$ in $(0, \infty)$. In constrast, $\lim _{\gamma \rightarrow 0^{+}} \Psi(\gamma)=+\infty$ and $\lim _{\gamma \rightarrow+\infty} \Psi(\gamma)=0$. Therefore, $\left(B_{1}\right)$ has only one solution.

If $\lambda+\mu=1 \quad(p+q=1), J(\gamma) \rightarrow 0$ as $\gamma \rightarrow+\infty$, it follows that $\Psi^{\prime}(\gamma)<0$ for $\gamma>0 . \Psi(\gamma) \rightarrow+\infty$ as $\gamma \rightarrow 0^{+}$and $\Psi(\gamma) \rightarrow 1$ as $\gamma \rightarrow+\infty$, however. Thus, there is no solution if $\delta \leq 1 \quad\left(a \leq 2^{1-p}\right)$, and there is a unique solution if $\delta>1 \quad\left(a>2^{1-p}\right)$.

If $\lambda+\mu>1(p+q>1), J(\gamma) \sim 2(\lambda+\mu-1)>0$ as $\gamma \rightarrow+\infty$, but $J(\gamma) \rightarrow-\infty$ as $\gamma \rightarrow 0^{+} . \Psi^{\prime}(\gamma)$ thus has only one sign change, and $\Psi(\gamma)$ decreases before increasing with $\lim _{\gamma \rightarrow 0^{+}} \Psi(\gamma)=+\infty$ and $\lim _{\gamma \rightarrow+\infty} \Psi(\gamma)=+\infty$. For this reason, $\left(B_{1}\right)$ has none, one, or two positive solutions according to whether $\varepsilon<\varepsilon_{c}, \varepsilon=\varepsilon_{c}$, or $\varepsilon>\varepsilon_{c}$, where $\varepsilon_{c}=\left(\Psi_{m}\right)^{\frac{2-p}{1-p-q}} 2^{\frac{1-p}{1-p-q}} a^{\frac{1}{p+q-1}}$.

The solution diagrams then have the form indicated in Figs. 1.1 and 1.2.

Next, for $\left(\mathrm{B}_{1}\right)$ with $g(u, v)=a u^{p} v^{q}+\frac{1}{2} \varepsilon u^{2}$, we have the equation

$$
\alpha^{p-2}=\frac{\varepsilon}{2 a}\left(2-e^{\varepsilon \beta}\right)\left(e^{\varepsilon \beta}-1\right)^{-1} \beta^{-q} \text {. }
$$


Setting $\left(2-e^{\varepsilon \beta}\right)\left(e^{\varepsilon \beta}-1\right)^{-1}=\gamma^{2}$ leads to $\beta=\frac{1}{\varepsilon} \ln \left(\frac{\gamma^{2}+2}{\gamma^{2}+1}\right)$. Define

$$
\Psi(\gamma)=\gamma^{\frac{2}{2-p}-1} \tan ^{-1} \frac{1}{\gamma}\left[\ln \left(\frac{\gamma^{2}+2}{\gamma^{2}+1}\right)\right]^{\frac{q}{p-2}}=\delta \quad \text { on }(0, \infty) .
$$

For $p>2$, calculations show that $\Psi^{\prime}(\gamma)<0, \Psi\left(0^{+}\right)=+\infty$, and $\Psi(+\infty)=0$. There is thus a unique solution. The same result holds for $p=2$ because the equation $Q(\beta)=\frac{2 a}{\varepsilon} \beta^{q}=\left(2-e^{\varepsilon \beta}\right)\left(e^{\varepsilon \beta}-1\right)^{-1}=R(\beta)$ has exactly one solution $\left(Q^{\prime}>0, Q\left(0^{+}\right)=0, Q(\infty)=+\infty ; R^{\prime}<0, R\left(0^{+}\right)=+\infty, R(\infty)=-1\right)$.

If $1 \leq p<2$, we have, with $\lambda=\frac{1}{2-p}, \mu=\frac{q}{2-p}$,

$$
\Psi(\gamma)=\gamma^{2 \lambda-1} \tan ^{-1} \frac{1}{\gamma}\left[\ln \left(\frac{\gamma^{2}+2}{\gamma^{2}+1}\right)\right]^{-\mu}
$$

and

$$
\Psi^{\prime}(\gamma)=\gamma^{2 \lambda}\left(\gamma^{2}+1\right)^{-1} \tan ^{-1} \frac{1}{\gamma}\left[\ln \left(\frac{\gamma^{2}+2}{\gamma^{2}+1}\right)\right]^{-\mu} J(\gamma),
$$

where

$$
J(\gamma)=\frac{(2 \lambda-1)\left(\gamma^{2}+1\right)}{\gamma^{2}}-\frac{1}{\left(\gamma \tan ^{-1} \frac{1}{\gamma}\right)}+2 \mu\left[\left(\gamma^{2}+2\right) \ln \left(\frac{\gamma^{2}+2}{\gamma^{2}+1}\right)\right]^{-1} .
$$

Note that $2 \lambda-1 \geq 1$. From the inequality $\left(\gamma^{2}+1\right) \tan ^{-1} \frac{1}{\gamma} \geq \gamma$, we see that $J(\gamma)>0$. It follows that $\Psi^{\prime}(\gamma)>0$ on $(0, \infty)$. Since $\Psi(0)=0$ and $\Psi(+\infty)=+\infty$, there is exactly one positive solution.

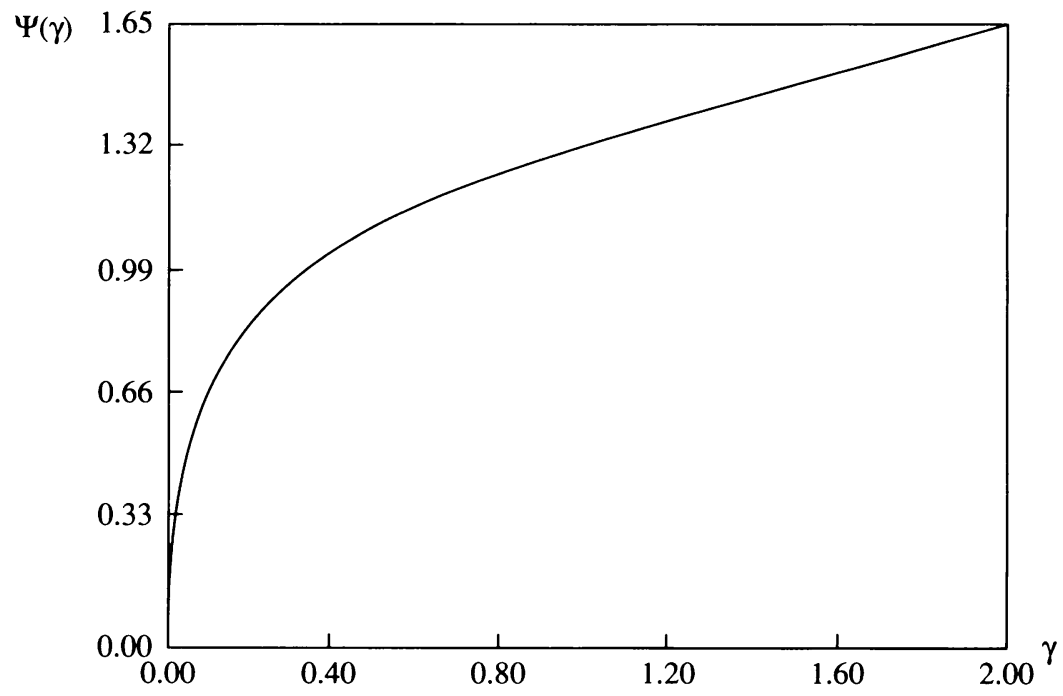

FIG. 0.1. $\Psi(\gamma)$ for $g(u, v)=a u^{p} v^{q}+\frac{1}{2} \varepsilon u^{2}, a>0, p=0.6, q=0.8$. 


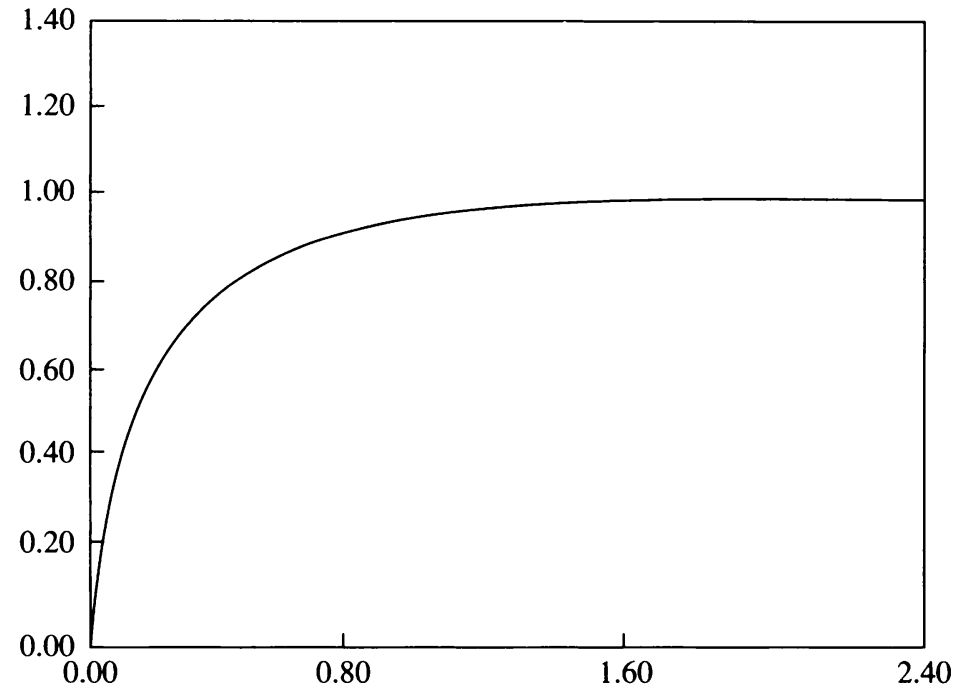

FIG. 0.2. $\Psi(\gamma)$ for $g(u, v)=a u^{p} v^{q}+\frac{1}{2} \varepsilon u^{2}, a>0, p=0.7, q=0.3$.

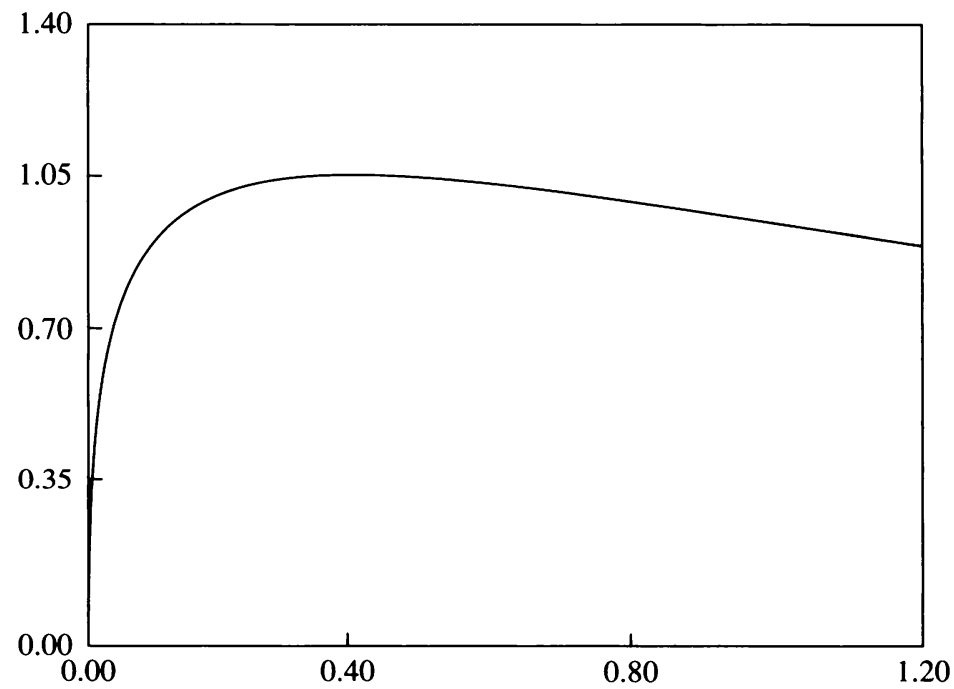

Fig. 0.3. $\Psi(\gamma)$ for $g(u, v)=a u^{p} v^{q}+\frac{1}{2} \varepsilon u^{2}, a>0, p=0.4, q=0.4$.

Although we cannot provide a rigorous analysis in case $0<p<1$, numerical results (see Fig. 0.1 - Fig. 0.3) support the following conjectures:

i) When $p+q>1, \Psi(0)=0, \Psi(+\infty)=+\infty$, one solution may be obtained.

ii) When $p+q=1, \Psi(0)=0, \lim _{\gamma \rightarrow+\infty} \Psi(\gamma)=1$, no solution may be obtained if $\delta \geq 1$, and one solution may be obtained if $\delta<1$.

iii) When $p+q<1, \Psi(0)=\Psi(+\infty)=0$ indicates that none, one, or two solutions may' be obtained.

Solution diagrams based, in part, on the numerical experiments are given in Figs. 2.1 and 2.2. 
Finally, for $g(u, v)=a u^{p} v^{q}$ or $g(u, v)=a u^{p} v^{q}+\frac{1}{2} \varepsilon u^{2}$, if $a<0$, from the condition $g(\alpha, \beta)-f(\alpha)>0$ in Theorem 2.4, we see that no positive solution can exist. Hence $w(x)$ must be trivial.

3. Comparison theorem and blow up of solutions. For simplicity, let $D_{T}=(0,1) \times$ $(0, T)$ and $D_{T} \cup \Gamma_{T}=[0,1] \times[0, T)$. We first define subsolution and supersolution of $(\mathrm{B})$.

Definition. A function $u(x, t)$ is called a subsolution of (B) on $D_{T}$ if $u \in$ $C^{2,1}\left(D_{T}\right) \cap C\left(D_{T} \cup \Gamma_{T}\right)$, satisfying

$$
\begin{aligned}
& u_{t} \leq u_{x x}+(f(u))_{x}, \quad 0<x<1, \quad 0<t<T, \\
& -u_{x}(0, t) \leq g(u,(0, t), \bar{u}), \quad 0<t<T, \\
& u(1, t) \leq 0, \quad 0<t<T, \\
& u(x, 0) \leq u_{0}(x), \quad 0 \leq x \leq 1 .
\end{aligned}
$$

A supersolution is defined by $\left(\mathbf{B}^{\prime}\right)$ with each " $\leq$ " replaced by " $\geq$ ".

By a similar argument as in [2], we then establish the comparison principle for problem (B).

TheOREM 3.1. Suppose that $f$ is continuously differentiable, $g_{\xi}(\xi, \eta)$ is continuous for $\xi \geq 0, \eta \geq 0$, and $g_{\eta}(\xi, \eta) \geq 0$ for $\xi \geq 0, \eta \geq 0$. Let $u$ be a nonnegative supersolution and $v$ a nonnegative subsolution of (B). Then $u \geq v$ in $D_{T} \cup \Gamma_{T}$.

We also obtain the following monotonicity result:

Corollary 3.2. If $u_{0}(x) \geq 0$ and $u(x, t) \geq u_{0}(x) \quad\left(\leq u_{0}(x)\right)$ in $D_{T} \cup \Gamma_{T}$ for (B), then $u_{t}(x, t) \geq 0(\leq 0)$ in $D_{T}$.

Sometimes the condition above is not easy to check. By requiring another restriction on the initial datum, we have the same conclusion.

Corollary 3.3. Suppose that the hypotheses for $f$ and $g$ in Theorem 3.1 are satisfied. If $u_{0}^{\prime \prime}+\left(f\left(u_{0}\right)\right)^{\prime} \geq 0(\leq 0)$ on $[0,1],-u_{0}^{\prime}(0)=g\left(u_{0}(0), \bar{u}_{0}\right)$, and $u_{0}(1)=0$, then $u_{t}(x, t) \geq 0(\leq 0)$ in $D_{T}$.

Following the same procedure as in [1], we can prove the local existence of solutions of (B). However, for certain nonlinearities and initial data, solutions of (B) blow up in finite time.

LEMMA 3.4. Let $u(x, t)$ be a nonnegative solution of (B) with $u_{x}(x, 0) \leq 0$, $g(\xi, \eta) \geq 0$ for $\xi, \eta \geq 0$. Then $u_{x}(x, t) \leq 0$ in $D_{T}$.

Theorem 3.5. Suppose that all hypotheses in Theorem 3.1 are satisfied and that $g(\xi, \eta) \geq 0$ for $\xi, \eta \geq 0$. Define $G(\xi, \eta)=\int_{0}^{\xi} g(s, \eta) d s, F(\xi)=\int_{0}^{\xi} f(s) d s$; and assume that $Q(\xi, \eta) \geq 0$ for some $\alpha>0$ and $\xi \geq 0, \eta \geq k>0$, where

$$
Q(\xi, \eta)=\xi g(\xi, \eta)-2(\alpha+1) G(\xi, \eta)+F(\xi)-\xi f(\xi) .
$$


Let $u$ be a solution of $(\mathrm{B})$ with $u_{0}(x) \geq v_{0}(x)$ on $[0,1]$. Here, $v_{0}(x)$ satisfies

$$
\begin{gathered}
v_{0}(x) \geq 0, \quad v_{0}^{\prime}(x) \leq 0, \quad v_{0}(1)=0, \quad v_{0}^{\prime \prime}(x)+\left(f\left(v_{0}\right)\right)^{\prime} \geq 0, \\
\text { and } \quad \int_{0}^{1} v_{0}(x) d x \geq k ; \\
-v_{0}^{\prime}(0)=g\left(v_{0}(0), \bar{v}_{0}\right), \\
\frac{1}{2} \int_{0}^{1} v_{0}^{\prime 2} d x<G\left(v_{0}(0), \bar{v}_{0}\right) .
\end{gathered}
$$

Then, $u$ will become unbounded in finite time.

Proof. Let $v(x, t)$ be the solution of (B) with $v(x, 0)=v_{0}(x)$; it is clear that $v_{t} \geq 0$ and $v_{x} \leq 0$ in $D_{T}$. We still define the same function $J(t)$ as in Theorem 3.7 of [1], but this time it leads to

$$
\begin{aligned}
J^{\prime \prime}(t)= & -2 v(0, t) f(v)+2 F(v)+2 v(0, t) g(v, \bar{v})-2 \int_{0}^{1} v_{x}^{2} d x+2 \beta \\
= & 2 Q(v(0, t), \bar{v})-4(\alpha+1) \int_{0}^{t} \int_{0}^{1} v_{x} v_{\eta} f^{\prime}(v) d x d \eta \\
& +4(\alpha+1)\left[\int_{0}^{t} \int_{0}^{1} v_{\eta}^{2} d x d \eta+\beta\right] \\
& +2\left[-2 \int_{0}^{t} v_{\eta}(0, \eta) g(v, \bar{v}) d \eta+G(v(0, t), \bar{v})-\int_{0}^{1} v_{0}^{\prime 2} d x\right. \\
& \left.-2 \alpha \int_{0}^{t} \int_{0}^{1} v_{\eta} v_{x x} d x d \eta-(2 \alpha+1) \beta\right] \\
\geq & 2 Q(v(0, t), \bar{v})+4(\alpha+1)\left[\int_{0}^{t} \int_{0}^{1} v_{\eta}^{2} d x d \eta+\beta\right] \\
& +2(\alpha+1)\left[G\left(v_{0}(0), v_{0}\right)-\frac{1}{2} \int_{0}^{1} v_{0}^{\prime 2} d x-\frac{(2 \alpha+1)}{2(\alpha+1)} \beta\right] .
\end{aligned}
$$

Then, combining (3.1), (3.2), and (3.4) yields the desired result.

As an application, take $f(\xi)=\frac{1}{2} \varepsilon \xi^{2}, g(\xi, \eta)=a \xi^{p} \eta^{q} \quad(p \geq 2, q>0)$, and $v_{0}(x)=A(1-x)^{r} \quad(A \geq 1, r \geq 3)$.

In view of the comparison theorem, we need to consider only the case $p=2$.

Conditions (3.3) and (3.4) require that $r>\frac{2}{3-p}=2$ and $A=\left[\frac{r(r+1)^{q}}{a}\right]^{1 /(q+1)}$. Additionally, we have to check $v_{0}^{\prime \prime}(x)+\varepsilon v_{0}(x) v_{0}^{\prime}(x) \geq 0$, which implies that $(r-1) \geq$ $\varepsilon A=\varepsilon\left[\frac{r(r+1)^{q}}{a}\right]^{1 /(q+1)}$, that is, $(r-1)^{q+1} \geq \varepsilon^{q+1} \frac{r(r+1)^{q}}{a}$. Since $r \geq 3$, it follows that $2(r-1) \geq r+1$; thus, it suffices to require $(r-1)^{q+1}>\varepsilon^{q+1} \frac{2^{q+1}(r-1)^{q+1}}{a}$. Consequently, we find

$$
a>(2 \varepsilon)^{q+1} .
$$

Then let $k=\int_{0}^{1} v_{0}(x) d x=\frac{A}{r+1}=\left[\frac{r}{a(r+1)}\right]^{1 /(q+1)}$. In order to check (3.1), we see 
that the following should be valid:

$$
a\left(1-\frac{2}{3}(\alpha+1)\right) \xi^{3} k^{q}-\frac{1}{3} \varepsilon \xi^{3} \geq 0 \text { for } \xi \geq 0,
$$

which can be done if $a k^{q}>\varepsilon$, that is, $a\left[\frac{r}{a(r+1)}\right]^{q /(q+1)}>\varepsilon, a>\varepsilon^{q+1}\left(1+\frac{1}{r}\right)^{q}$; this is automatically satisfied if $(3.5)$ holds.

For problem (B) with $g(\xi, \eta)=a \xi^{p} \eta^{q}+\frac{1}{2} \varepsilon \xi^{2}$ and $u_{0} \geq v_{0}$, the solution blows up because the solution of (B) with $g(\xi, \eta)=a \xi^{p} \eta^{q}$ is a subsolution.

4. Stability and instability analysis. In this section, we shall study the large time behavior of global solutions. We use two different approaches to build up the criteria for stability and instability.

First, we state a lemma, whose proof is similar to that for Theorem 3.2A in [3] and hence is omitted.

LEMMA 4.1. Let $u(x, t)$ be a bounded monotone solution of (B); then $u(x, t)$ exists for all time and tends to a stationary solution of $(B)$.

Now with somewhat weak assumptions, we can show one stability result:

Theorem 4.2. Let all conditions in Theorem 2.3 be satisfied, and let $w(x)$ be the corresponding stationary solution of $(\mathbf{B})$. Then $w(x)$ is stable.

Proof. By Theorem 2.3, we know that $w(x)$ is the unique positive solution of $\left(\mathrm{B}_{1}\right)$. Setting $v(x)=\left(1+\delta_{0}\right) w(x) \quad\left(\delta_{0}>0\right)$, we have

$$
\begin{aligned}
v_{x x}+f^{\prime}(v) v_{x} & =\left(1+\delta_{0}\right)\left[w_{x x}+f^{\prime}\left(\left(1+\delta_{0}\right) w\right) w_{x}\right] \\
& \leq\left(1+\delta_{0}\right)\left[w_{x x}+f^{\prime}(w) w_{x}\right] \\
& \leq 0 .
\end{aligned}
$$

Moreover, at $x=0$,

$$
\begin{aligned}
-v_{x}-g(v, \bar{v}) & =\left(1+\delta_{0}\right) g(w, \bar{w})-g\left(\left(1+\delta_{0}\right) w,\left(1+\delta_{0}\right) \bar{w}\right) \\
& =\left(1+\delta_{0}\right) w\left[g(w, \bar{w}) / w-g\left(\left(1+\delta_{0}\right) w,\left(1+\delta_{0}\right) \bar{w}\right) /\left(1+\delta_{0}\right) w\right] \\
& \geq 0 .
\end{aligned}
$$

Hence, $v(x)$ is a supersolution, and it follows that any solution $u_{\delta}(x, t)$ of $(\mathrm{B})$ with $u_{\delta}(x, 0)=(1+\delta) w(x) \quad\left(0<\delta<\delta_{0}\right)$ is bounded away from $v(x)$ and monotonically increasing as $\delta \rightarrow \delta_{0}^{-}$. Therefore, the solution $u(x, t)$ with $u(x, 0)=\left(1+\delta_{0}\right) w(x)$ satisfies $w(x) \leq u(x, t) \leq v(x)$, and $u_{t} \leq 0$ in $D_{T}$ as Corollary 3.2 is applied. The preceding lemma shows that $u(x, t)$ goes to $w(x)$ as $t \rightarrow \infty$, which implies that $w(x)$ is stable from above. Using $u(x, t)$ with $u(x, 0)=\left(1-\delta_{1}\right) w(x)$, we can show that $w(x)$ is stable from below.

Applying a few more conditions to $f$ and $g$, we can present a more complete result for stability. For this purpose, we replace $f$ by $\varepsilon f$ and then denote the stationary solution $w(x)$ by $w(x, \varepsilon)$.

ThEOREM 4.3. Assume that $\mathrm{f}$ is twice continuously differentiable, $f^{\prime}(\xi)>0$ and $f^{\prime \prime}(\xi) \geq 0$ for $\xi>0, g_{\xi}(\xi, \eta)$ is continuous for $\xi \geq 0, \eta \geq 0$, and $g_{\eta}(\xi, \eta) \geq 0$ 
for $\xi, \eta \geq 0$. Let $w(x, \varepsilon)$ be a $C^{1}$ (in $\varepsilon$ ) positive stationary solution for $a \leq \varepsilon \leq b$, and let $w_{0}(\varepsilon)=w(0, \varepsilon)$. Then if $w_{0}^{\prime}(\varepsilon) \leq 0$, the solutions are stable; whereas they are unstable if $w_{0}^{\prime}(\varepsilon)>0$ on $[a, b]$.

The proof is actually the same as that for Theorem 3.6B in [3] and is therefore omitted.

By means of the above theorem, we give criteria on stability and draw the solution diagrams. For $\left(\mathrm{B}_{1}\right)$ with $g(\xi, \eta)=a \xi^{p} \eta^{q}$, we first observe that

$$
\varepsilon=2 w_{0}^{-1}(\varepsilon) \gamma^{-1} \tan ^{-1} \frac{1}{\gamma}
$$

Substituting (4.1) into (2.1), we find that

$$
\left[w_{0}(\varepsilon)\right]^{\frac{p+1-1}{1+q}}=\left(\frac{2^{q}}{a}\right)^{\frac{1}{1+q}} \tan ^{-1} \frac{1}{\gamma} \gamma^{-1}\left(\gamma^{2}+1\right)^{\frac{1}{1+q}}\left[\ln \left(\frac{\gamma^{2}+2}{\gamma^{2}+1}\right)\right]^{-\frac{q}{1+q}} .
$$

If $p+q \neq 1,(4.2)$ can be rewritten as

$$
\left[w_{0}(\varepsilon)\right]^{\frac{p+q-1}{1+q}}=\left(\frac{2^{q}}{a}\right)^{\frac{1}{1+q}} \Psi(\gamma) .
$$

Here, $\Psi(\gamma)$ is defined as in (2.5), with $\lambda=\frac{1}{1+q}, \mu=\frac{q}{1+q}$. Similarly,

$$
\left(\frac{p+q-1}{1+q}\right)\left[w_{0}(\varepsilon)\right]^{\frac{p-2}{1+q}} w_{0}^{\prime}(\varepsilon)=\left(\frac{2^{q}}{a}\right)^{\frac{1}{1+q}} \Psi^{\prime}(\gamma) \gamma^{\prime}(\varepsilon) .
$$

Because $\Psi^{\prime}(\gamma)<0$ for $\gamma>0$, then if $p+q>1, w_{0}^{\prime}(\varepsilon)$ has the opposite sign to $\gamma^{\prime}(\varepsilon)$; but if $p+q<1$, it has the same sign.

If $p=2$, by $(2.1)$, we obtain $\varepsilon^{1+q}=\psi(\gamma)=2 a\left(\gamma^{2}+1\right)^{-1}\left[\ln \left(\frac{\gamma^{2}+2}{\gamma^{2}+1}\right)\right]^{q}$ and find $\gamma^{\prime}(\varepsilon)<0$; hence, $w_{0}^{\prime}(\varepsilon)>0$.

For $p>2$, the fact that $\Psi^{\prime}(\gamma)<0$ leads to $\gamma^{\prime}(\varepsilon)<0$. But for $1 \leq p<2$ or $0<p<1 \quad(p+q>1)$, we can see that $\Psi^{\prime}(\gamma)$ changes sign once from negative to positive. Therefore, there exist two branches: $\gamma^{\prime}(\varepsilon)>0$ for one, and $\gamma^{\prime}(\varepsilon)<0$ for the other.

Then, for the case $p+q<1$, the result $\gamma^{\prime}(\varepsilon)<0$ follows from $\Psi^{\prime}(\gamma)<0$.

At last, we discuss $p+q=1$. Obviously, $\gamma^{\prime}(\varepsilon)=0$. Noticing (4.1), we see that $w_{0}^{\prime}(\varepsilon)<0$.

In summary, for $p \geq 2, w(x, \varepsilon)$ is unstable; whereas it is stable for $p+q \leq 1$. For $1 \leq p<2$ or $0<p<1 \quad(p+q>1)$, there are two branches-one stable but the other unstable.

Then, using (2.4) and (4.2), we can carry out a discussion on $w_{0}(\varepsilon)$ graphically.

For $p>2$ and $p+q<1, \varepsilon \rightarrow 0$ if $\gamma \rightarrow \infty$ and $\varepsilon \rightarrow+\infty$ as $\gamma \rightarrow 0^{+}$. Therefore, $w_{0}(0)=\left(\frac{2^{q}}{a}\right)^{\frac{1}{p+q-1}}$ for both cases. However, $\lim _{\varepsilon \rightarrow+\infty} w_{0}(\varepsilon)=+\infty$ for $p>2$; whereas $\lim _{\varepsilon \rightarrow+\infty} w_{0}(\varepsilon)=0$ if $p+q<1$. The case $p=2$ needs to be treated separately because now $\varepsilon$ is bounded by $\varepsilon_{2}=(2 a)^{\frac{1}{1+q}}(\ln 2)^{\frac{q}{1+q}}$, and $\varepsilon^{1+q}=\psi(\gamma)$ implies that $\varepsilon \rightarrow \varepsilon_{2}$ as $\gamma \rightarrow 0^{+}$. Thus, $w_{0}(0)=\left(\frac{2^{q}}{a}\right)^{\frac{1}{p+q-1}}$ and $\lim _{\varepsilon \rightarrow \varepsilon_{2}^{-}} w_{0}(\varepsilon)=+\infty$. 


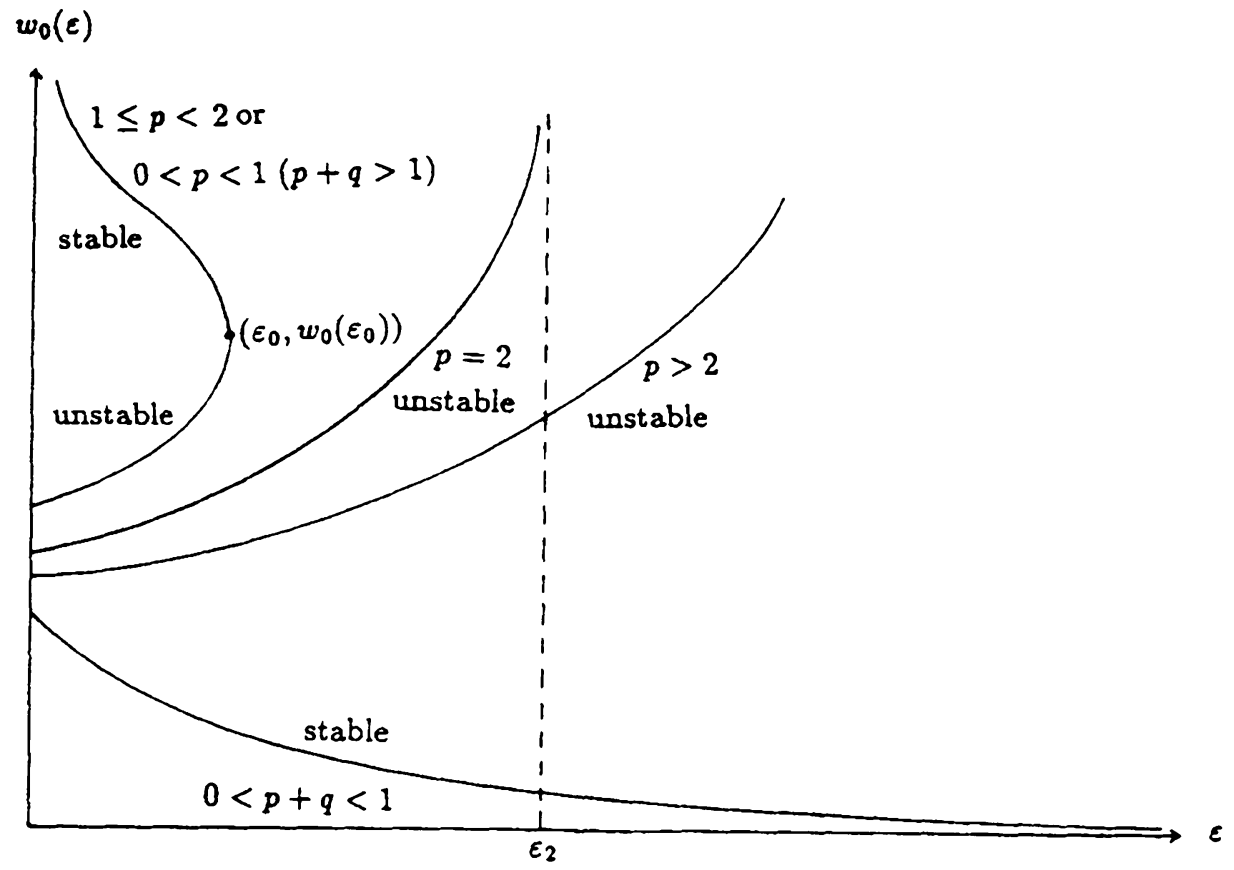

FIG. 1.1. $w_{0}(\varepsilon)$ for $g(\xi, \eta)=a \xi^{p} \eta^{q}, 0<a \leq 2^{1-p}$ and $w_{0}(0)=$ $\left(2^{q} / a\right)^{1 /(p+q-1)}$

For $1 \leq p<2$ and $0<p<1(p+q>1), \varepsilon$ runs from 0 to $\varepsilon_{0}<\infty$, since $\Psi \geq \Psi_{m}$ on $(0, \infty)$. For this reason, the unstable branch of $w(x, \varepsilon)$ increases from $\left(\frac{2^{q}}{a}\right)^{\frac{1}{p+q-1}}$ to $w_{0}\left(\varepsilon_{0}\right)$; whereas the stable one decreases from infinity to $w_{0}\left(\varepsilon_{0}\right)$.

Finally, for $p+q=1$, note that $\gamma^{\prime}(\varepsilon)=0$ and (4.1); so we find that $\lim _{\varepsilon \rightarrow 0^{+}} w_{0}(\varepsilon)$ $=+\infty$ and $\lim _{\varepsilon \rightarrow+\infty} w_{0}(\varepsilon)=0$ if $2^{1-p}<a$.

The solution diagrams are given in Figs. 1.1 and 1.2.

Next, for $\left(\mathrm{B}_{1}\right)$ with $g(\xi, \eta)=a \xi^{p} \eta^{q}+\frac{1}{2} \varepsilon \xi^{2}$, we observe that the equation for $\varepsilon$ is the same as (4.1). Substituting (4.1) into (2.6), we find that

$$
\left[w_{0}(\varepsilon)\right]^{\frac{p+q-1}{1+q}}=\left(\frac{2^{q}}{a}\right)^{\frac{1}{1+q}} \tan ^{-1} \frac{1}{\gamma} \gamma^{\frac{1-q}{1+q}}\left[\ln \left(\frac{\gamma^{2}+2}{\gamma^{2}+1}\right)\right]^{-\frac{q}{1+q}} \text {. }
$$

If $p+q \neq 1,(4.1)$ can be rewritten as

$$
\left[w_{0}(\varepsilon)\right]^{\frac{p+q-1}{1+q}}=\left(\frac{2^{q}}{a}\right)^{\frac{1}{1+q}} \Psi(\gamma) .
$$

Here, $\Psi(\gamma)$ is defined as in (2.8), with $\lambda=\frac{1}{1+q}, \mu=\frac{q}{1+q}$. Similarly,

$$
\left(\frac{p+q-1}{1+q}\right)\left[w_{0}(\varepsilon)\right]^{\frac{p-2}{1+q}} w_{0}^{\prime}(\varepsilon)=\left(\frac{2^{q}}{a}\right)^{\frac{1}{1+q}} \Psi^{\prime}(\gamma) \gamma^{\prime}(\varepsilon) .
$$

Suppose $\Phi^{\prime}(\gamma)>0$ for $\gamma>0$, an assumption supported by numerical computation; then if $p+q<1, w_{0}^{\prime}(\varepsilon)$ has the opposite sign to $\gamma^{\prime}(\varepsilon)$; but if $p+q>1$, it has the same sign. 


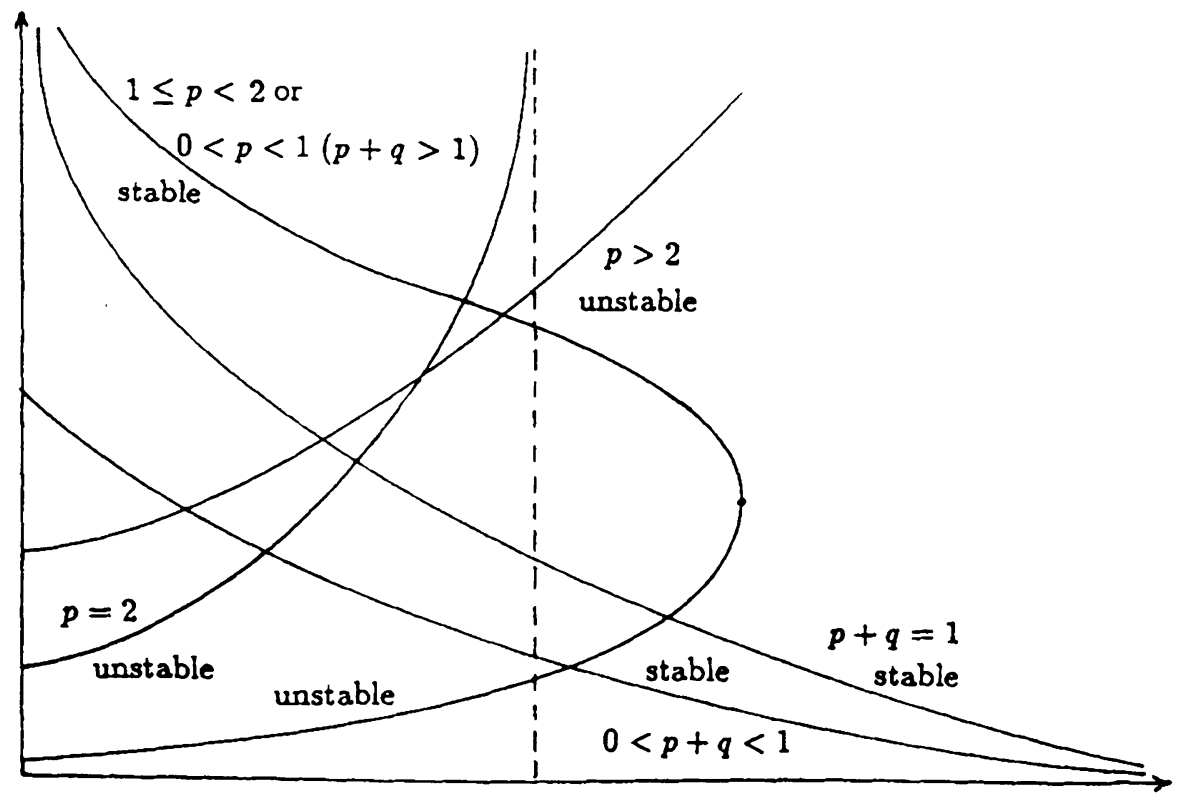

Fig. 1.2. $w_{0}(\varepsilon)$ for $g(\xi, \eta)=a \xi^{p} \eta^{q}, a>2^{1-p}$ and $w_{0}(0)=$ $\left(2^{q} / a\right)^{1 /(p+q-1)}$ 0 .

If $p=2$, by $(2.6)$, we obtain $\varepsilon^{1+q}=\psi(\gamma)=2 a \gamma^{-2}\left[\ln \left(\frac{\gamma^{2}+2}{\gamma^{2}+1}\right)\right]^{q}$ and find $\gamma^{\prime}(\varepsilon)<$

For $p>2$, the fact that $\Psi^{\prime}(\gamma)<0$ leads to $\gamma^{\prime}(\varepsilon)<0$. The same holds for $1 \leq p<2$ and for $p<1 \quad(p+q>1)$ since $\Psi^{\prime}(\gamma)>0$.

Then for the case $p+q<1$, we can see that $\Psi^{\prime}(\gamma)$ changes sign once from positive to negative. Therefore, there exist two branches: $\gamma^{\prime}(\varepsilon)>0$ for one, and $\gamma^{\prime}(\varepsilon)<0$ for the other.

Ai last, we discuss $p+q=1$. Obviously, $\gamma^{\prime}(\varepsilon)=0$. Noticing (4.1), we see that $w_{0}^{\prime}(\varepsilon)<0$.

In summary, for $p \geq 1$ and $0<p<1 \quad(p+q \geq 1), w(x, \varepsilon)$ is stable; whereas for $0<p+q<1$, there are two branches - one stable but the other unstable.

The solution diagrams are given in Figs. 2.1 and 2.2 (see p. 566).

Finally, we turn our attention to the steady-state problem for Burgers's equation with $a<0$. First, consider problem (B) with $g(\xi, \eta)=\frac{1}{2} \varepsilon \xi^{2}$, whose solution and stationary solution may be denoted without confusion by $u_{B}(x, t, \varepsilon)$ and $w_{B}(x, \varepsilon)$, respectively. Then, the comparison theorem shows that the solution of $(B)$ with $g(\xi, \eta)=a \xi^{p} \eta^{q}$ or $g(\xi, \eta)=a \xi^{p} \eta^{q}+\frac{1}{2} \varepsilon \xi^{2}$ is bounded by $u_{B}(x, t, \varepsilon)$ if $u(x, 0) \leq$ $u_{B}(x, 0, \varepsilon)$.

Set $u_{B}(x, 0, \varepsilon)=w_{B}(x, \sigma)(\sigma<\varepsilon)$ to find

$$
\begin{aligned}
u_{B t}(x, 0, \varepsilon) & =w_{B}^{\prime \prime}+\varepsilon w_{B} w_{B}^{\prime} \\
& =w_{B}^{\prime \prime}+\sigma w_{B} w_{B}^{\prime}+(\varepsilon-\sigma) w_{B} w_{B}^{\prime} \\
& <0,
\end{aligned}
$$

since $w_{B}^{\prime}<0$. From this, we conclude as before, that $u_{B t}(x, t) \leq 0$ in $D_{T}$. Note 


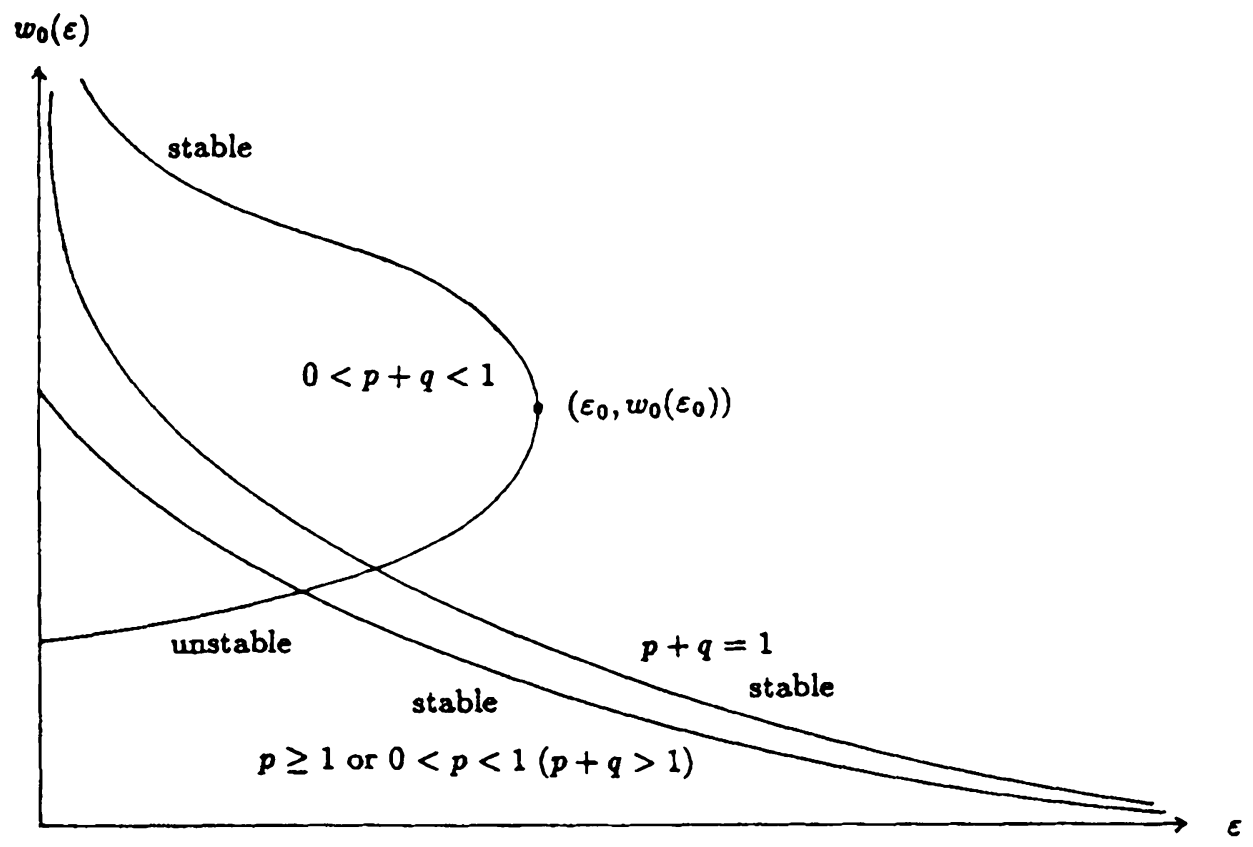

FIG. 2.1. $w_{0}(\varepsilon)$ for $g(\xi, \eta)=a \xi^{p} \eta^{q}+\frac{1}{2} \varepsilon \xi^{2}, 0<a<2^{1-p}$ and $w_{0}(0)=\left(2^{q} / a\right)^{1 /(p+q-1)}$

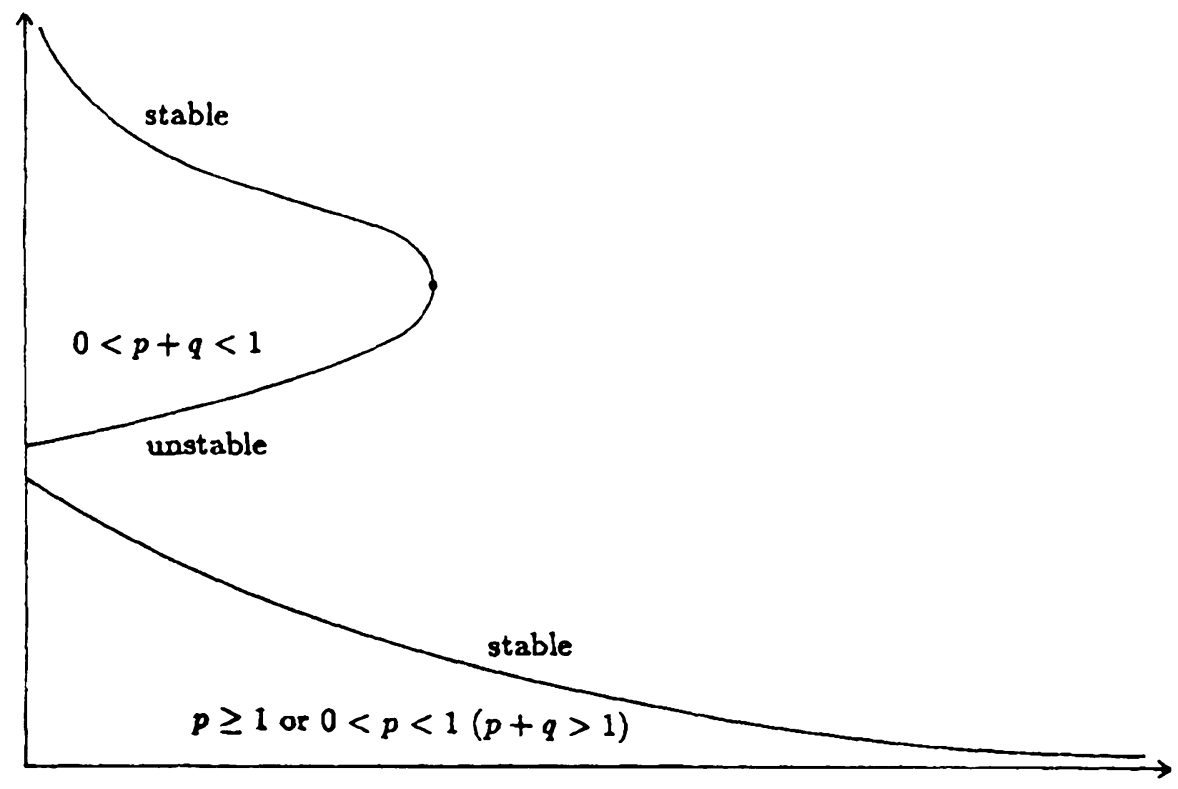

FIG. 2.2. $w_{0}(\varepsilon)$ for $g(\xi, \eta)=a \xi^{p} \eta^{q}+\frac{1}{2} \varepsilon \xi^{2}, a \geq 2^{1-p}$ and $w_{0}(0)=\left(2^{q} / a\right)^{1 /(p+q-1)}$

that $w_{B}(x, \sigma)<w_{B}(x, \varepsilon)$ in a neighborhood of $x=0$. Thus, $u_{B}(x, t)$, as well as $u(x, t)$, approaches zero as $t$ tends to infinity. This indicates that the null solution of $\left(B_{1}\right)$ is stable from above. 
Acknowledgments. This is part of the author's doctoral thesis, completed at Iowa State University under the direction of Professor H. A. Levine. The author wishes to express his deepest appreciation to Professor Levine for his valuable help and encouragement. The author would also like to thank Professor P. E. Sacks for many useful suggestions.

\section{REFERENCES}

[1] K. Deng, Behavior of solutions of Burgers' equation with nonlocal boundary conditions (I), J. Differential Equations (to appear)

[2] K. Deng, M. K. Kwong, and H. A. Levine, The influence of nonlocal nonlinearities on the long time behavior of solutions of Burgers's equation, Quart. Appl. Math. 50, 173-200 (1992)

[3] H. A. Levine, Stability and instability for solutions of Burgers' equation with a semilinear boundary condition, SIAM J. Math. Anal. 19, 312-336 (1988) 